\title{
La mejora de la productividad: ¿el santo grial de los sistemas de salud en el mundo?
}

\author{
RICARDO ARTURO VEGA RODRÍGUEZ \\ Politécnico Grancolombiano \\ rvegarod@poli.edu.co
}

Químico Farmacéutico y Magíster en Administración. Profesor de Mercadeo en el Politécnico Grancolombiano. Sus actividades de docencia e investigación se focalizan en las áreas de Mercadeo Farmacéutico y Estratégico.

\begin{abstract}
Resumen
Con base en una revisión de literatura nacional e internacional se presenta el concepto de productividad y su análisis por medio de la función de producción de Cobb-Douglas. Se aplican estos conceptos al área de salud y se muestran resultados logrados tanto en Colombia como en otros contextos. Posteriormente se expone la estructura del sistema de salud de Colombia y se hacen algunas recomendaciones de avance en las prácticas de gestión del sistema y así lograr la mejora de sus resultados productivos.
\end{abstract}

\section{Palabras Claves}

Productividad, factores de producción, función de producción de Cobb-Douglas, sistema de salud colombiano.

\section{Abstract}

The concept of productivity is presented based on a national and international literature review and on its analysis through the Cobb-Douglas production function. These concepts are applied to the health area and the results found not only in Colombia, but also in other contexts are displayed. Afterwards, the Colombian health system structure is shown and some recommendations in order to improve the management practices and productive results in this sector are given.

\section{Key words}

Productivity, factors of production, Cobb-Douglas production function, Colombian health system.
Fecha de recepción: 10 de septiembre de 2010 Fecha de aprobación: 30 de septiembre de 2010

\section{Introducción}

Los sistemas de salud en el mundo y en Colombia están enfrentados a grandes retos como son el de su sostenibilidad financiera, el de la mejora en los índices de satisfacción de sus usuarios y en la contención de los crecientes gastos en salud. En atención a esta problemática es pertinente reflexionar sobre la productividad de la industria de la salud para así poder proponer cambios en la gestión de la misma, en aras del progreso en los factores mencionados.

\section{Concepto de productividad}

Las organizaciones se enfrentan a diversos entornos competitivos que determinan el acceso a los diferentes factores productivos, como son la naturaleza, el recurso humano y el capital, generándoles restricciones en las posibilidades de combinación de los elementos citados. Esas combinaciones de las posibilidades factibles se engloban en el conjunto de producción y dan lugar a una función de producción, que es simplemente el volumen máximo generado y que es posible con una cantidad dada de factores y se constituye en la frontera de dicho conjunto (Varian, 1999).

Una función de producción ampliamente usada y referenciada internacionalmente es la de Cobb-Douglas, que toma la forma:

$$
\mathrm{f}\left(\mathrm{x}_{1}, \mathrm{x}_{2}\right)=\mathrm{Ax} 1^{\mathrm{a}} \mathrm{x} 2^{\mathrm{b}}
$$

o en su representación logarítmica

$$
\mathrm{f}^{\prime}\left(\mathrm{x}_{1}, \mathrm{x}_{2}\right)=\mathrm{a} \ln \mathrm{x}_{1}+\mathrm{b}_{1} \mathrm{n}_{2}
$$


La constante A mide la escala de producción cuando se utiliza una unidad de cada factor productivo y a y b la respuesta de la cantidad producida a las variaciones de los factores (Varian, 1999).

Se han realizado estudios de productividad en diversos sectores industriales del área de manufactura y se pueden citar ejemplos como los de la soya (Mingming y Dongmei, 2010), pero también del área de servicios, como es el caso de los fondos de pensiones (Pestana y Medeiros, 2007) y de la industria de las aerolíneas (Vasigh y Fleming, 2005), o bien el impacto que ha tenido internet en la productividad (Hornstein y Krusell, 2000).

Pero al igual que se realizan estudios de productividad por sectores industriales específicos también se encuentran trabajos en los que se analiza el comportamiento industrial de un país o región del mismo, como en el caso de investigaciones desarrolladas en Estados Unidos (Dolmas, Raj, y Slottje, 1999; Felipe y Adams, 2005; Vinod, 1973), Reino Unido (Geroski, Kretschmer, y Walters, 2009), China (Ozyurt, 2009) y Colombia (Eslava, Haltiwanger, Kugler, y Kugler, 2006; Posada, 1993; Sánchez, Rodríguez, y Núñez, 1996), o incluso los desarrollados en regiones como América Latina (Contreras y Gatica, 2009), Este Asiático (Felipe, 1999; Liao, Holmes, Weyman-Jones, y Llewellyn, 2007), o muestras internacionales de diferentes regiones (Cermeño y Vásquez, 2006; Islam, 2008; Stresing, Lindenberger, y Kümmel, 2008).

\section{Productividad en los sistemas de salud}

Como uno de los sectores de mayor impacto económico y social en nuestras sociedades, el de salud ha sido una de las áreas en la que la economía ha desarrollado, por medio de la economía de la salud, un robusto cuerpo teórico, teniendo en cuenta el tipo de bien que maneja y la alta sensibilidad de las personas tanto frente a la posesión del mismo como a su experiencia de consumo, cuando recurren a servicios de salud en sus componentes de aseguramiento, y prevención y tratamiento al buscar igualmente el aumento de productividad y la eficiencia de dichos servicios.

En Colombia se han realizado varios estudios que han analizado la situación de productividad en los hospitales e instituciones prestadoras de servicios (IPS) al valorar el impacto que el cambio del sistema de salud ha tenido sobre este tipo de organizaciones. Sarmiento, Castellanos, Nieto, Alonso y Pérez (2005) realizaron un análisis con la red pública de prestadores de servicios para evaluar la gestión en este sector y para lo cual usaron la función Cobb-Douglas argumentando que es de fácil estimación y que en la mayoría de estudios reporta resultados satisfactorios y acordes con la teoría económica. Las IPS analizadas fueron divididas de acuerdo con su nivel de complejidad en los tres niveles normalmente reconocidos.

En este trabajo se encontró una situación de déficit físico y humano en estas instituciones, aunque la estructura administrativa se constituye en un rubro de demasiado peso en su gasto de personal. Desde uno de los productos del sistema, como son los planes de promoción y prevención se detectó una atención deficitaria lo que conlleva a desprotección de sectores vulnerables de la población y al aumento de casos de alto riesgo como de muertes evitables. Otro producto evaluado fue el de la calidad de los servicios prestados, donde se lograron indicadores óptimos (Sarmiento, Castellanos, Nieto, Alonso y Pérez, 2005).

Mutis, Díz y Toro (2006) desarrollaron un estudio centrándose específicamente en los hospitales colombianos de nivel II y donde igualmente usaron la función de producción de Cobb-Douglas. Con una muestra de 113 hospitales llegaron a una jerarquización de los mismos y presentan los hospitales más eficientes e ineficientes de la muestra tomada.

Finalmente, Maldonado y Tamayo (2007) hicieron también un trabajo con los hospitales públicos colombianos en el periodo 2002-2005, usando la información proveniente del sistema de información hospitalario (siho). Concluyen que la medición de producción es una medida de proceso y no de resultados, debido a las variables usadas, pero puede ocurrir que un hospital aparentemente más eficiente lo esté logrando a costa del sacrificio de la calidad del servicio, con lo que podría estar generando mayores costos en el largo plazo al aumentar las complicaciones derivadas de la mala atención. Sugieren entonces afinar la información usada y dirigirla más al tema de los resultados que del proceso.

Wholey, Engberg y Bryce (2006) estudiaron la productividad de las Hmos (Health Maintenance Organizations) en Estados Unidos, en el periodo 1985 a 2001. Como medida primaria de producto se tomó la de meses-miembro totales de cobertura y como medidas de factores de producción se usaron las de cuidado hospitalario, visitas ambulatorias, recursos administrativos y otros recursos médicos. Los resultados mostraron ganancias de productividad en el periodo de 1990 a 
1996 y pérdidas en el de 1997 a 1999 debido, según los autores, a retrocesos presentados en la normatividad del sistema. Una de las causas que atribuyeron para la mejora de los índices de productividad es la de procesos de aprendizaje organizacional. Se argumenta también que la inclusión de los usuarios y asegurados en los procesos de toma de decisiones llevó a enriquecer la mezcla de insumos para la provisión de los servicios y la mejora de los resultados.

\section{El sistema de salud en Colombia}

En el país, al igual que en el resto del mundo, la salud representa uno de los sectores económicos más importantes y dinámicos y es así como el gasto nacional en salud representó en 2003 un 7,8\% del PIB y el gasto público per cápita en salud en el mismo año fue de 116 dólares (Ministerio de la Protección Social y Organización Panamericana de la Salud, 2008).

Pero la importancia económica y social de este sector no se complementa con la estabilidad financiera de los sistemas de salud, incluido el colombiano, como se evidenció en la reforma del sistema de salud aprobada en Estados Unidos en el primer semestre de 2010 (Samuelson, 2010) ni tampoco con la satisfacción que genera en los usuarios del sistema, como ocurre en Colombia (Losada y Rodríguez, 2007).

El sistema de salud colombiano está basado en el sistema anglosajón de prestación de servicios públicos, simulando un mercado competitivo (Chicaíza, 2002) con actores del Estado, sector privado y usuarios. El ente rector del sistema es la Comisión de Regulación en Salud (CRES), una unidad administrativa especial adscrita al Ministerio de la Protección Social (2010). Por parte del Estado, participan también en el sistema, el propio Ministerio de la Protección Social, la Superintendencia de Salud y el Fosyga (Fondo de Solidaridad y Garantía), organismo encargado de la administración de los recursos financieros del sistema.

En el sector privado participan las EPs (entidades promotoras de salud), empresas encargadas de administrar el esquema de aseguramiento, tanto en el régimen contributivo (personas con capacidad de pago) como en el subsidiado (personas sin capacidad de pago seleccionadas), y de la coordinación en la prestación de los servicios y en una proporción significativa la inte- gración vertical con IPS (instituciones prestadoras de servicios de salud) pertenecientes a los mismos grupos económicos. Además están las IPS, encargadas de proveer los servicios pactados con las EPS (Restrepo, Lopera y Rodríguez, 2007). Y la industria farmacéutica y de dispositivos médicos que provee con este tipo de productos al sistema. Es de anotar que las EPS e ips no son exclusivamente privadas, sino que existen algunas de régimen mixto (por ejemplo, la nueva EPS) y otras unicamente públicas.

En su informe correspondiente a 2009, Acemi (Asociación Colombiana de Empresas de Medicina Integral) destaca que el 91,3\% de la población colombiana está afiliada al sistema general de seguridad social en salud, de los cuales el 40,2\% está en el régimen contributivo y un $51,2 \%$ en el subsidiado. Sus agremiados reúnen el $44,4 \%$ de la población afiliada, cubriendo el 86,5\% de los contributivos y $11,3 \%$ de los subsidiados. Acemi cuenta con catorce EPS afiliadas, de las cuales doce son del régimen contributivo y dos del subsidiado. Se encuentran empresas propiedad de grupos económicos privados, del sector solidario y de cajas de compensación (Acemi, 2009).

La población restante del régimen contributivo está cubierta por la Nueva EPS, que surgió a raíz de la semiprivatización de la EPS estatal, Instituto de los Seguros Sociales (Iss) y que quedó conformada por la unión de seis cajas de compensación, que poseen la mayoría accionaria y la empresa industrial y comercial del Estado, Compañía de Seguros Positiva y la cual quedó como socio minoritario (Nueva EPS, 2010).

\section{Discusión}

A partir del marco de referencia desarrollado en los párrafos previos se pueden recomendar los siguientes elementos de gestión que apunten a mejorar los resultados del sistema de salud colombiano.

1. Realizar estudios amplios y longitudinales que cobijen a todos los actores del sistema y que permitan definir de manera confiable cuáles son los factores de productividad más influyentes en la industria de salud colombiana, con el fin de poder actuar sobre ellos e impactar los resultados del sistema de una manera significativa. 
2. Generar más información que cubra los resultados "duros" del sistema como son los financieros, de eficiencia económica, pero sobre todo los de satisfacción de los usuarios pensando en conocer cuáles son las variables que ayudarían a mejorar desde la perspectiva del cliente, su nivel de satisfacción con el sistema. Esto contrastaría con la situación actual, donde muchas de las medidas se refieren a resultados intermedios o "blandos" del sistema.

3. Incluir a los usuarios en los órganos de toma de decisiones por medio de todo el sistema para que puedan aportar, con sus percepciones y necesidades como consumidores, a mejorar los procesos decisorios del sistema.

4. En general, se deben fortalecer las prácticas de gestión, tanto públicas como privadas, en el sistema de salud colombiano, para que ayuden a mejorar la productividad del sistema y, finalmente, contribuyan a la viabilidad financiera del mismo y aumento de la satisfacción de los usuarios. Uno de los puntos que merece un enfoque especial es el de la gestión de la información para que la toma de decisiones se lleve de una manera racional e informada.

\section{Bibliografía}

1. Asociación Colombiana de Empresas de Medicina Integral (Acemi). (2009). Acemi - Asociación Colombiana de Empresas de Medicina Integral. Consultado el 12 de septiembre de 2010, de http://www.acemi. org.co/index.html

2. Cermeño, R., \& Vásquez, S. (2006). Tecnología Agrícola Internacional Estudio comparativo con modelos panel no lineales. Trimestre Económico, 73(291), 539-573.

3. Chicaíza, L. (2002). El mercado de la salud en Colombia y la problemática del alto costo. Problemas del Desarrollo, 33(131), 163-187.

4. Contreras, R., \& Gatica, L. (2009). Factores productivos y crecimiento económico: una función de producción para América Latina. Revista Chilena de Economía y Sociedad, 3(1), 55-67.
5. Dolmas, J., Raj, B., \& Slottje, D. (1999). The U.S. Productivity Slowdown: A Peak Through the Structural Break Window. Economic Inquiry, 37(2), 226-241.

6. Eslava, M., Haltiwanger, J., Kugler, A., \& Kugler, M. (2006). Plant Turnover and Structural Reforms in Colombia. IMF Staff Papers, 53(Special Issue), 58-75.

7. Felipe, J. (1999). Total Factor Productivity Growth in East Asia: A Critical Survey. Journal of Development Studies, 35(4), 1-41.

8. Felipe, J., \& Adams, G. (2005). 'A Theory of Production': The Estimation of the Cobb-Douglas Function: A Retrospective View. Eastern Economic Journal, 31(3), 427-445.

9. Geroski, P., Kretschmer, T., \& Walters, C. (2009). Corporate Productivity Growth: Champions, Leaders, and Laggards. Economic Inquiry, 47(1), 1-17.

10. Hornstein, A., \& Krusell, P. (2000). The IT Revolution: Is It Evident in the Productivity Numbers? Economic Quarterly, 86(4), 49-78.

11. Islam, N. (2008). Determinants of Productivity across Countries: An Exploratory Analysis . Journal of Developing Areas, 42(1), 201-242.

12. Liao, H., Holmes, M., Weyman-Jones, T., \& Llewellyn, D. (2007). Productivity growth of East Asia economies' manufacturing: A decomposition analysis. Journal of Development Studies, 43(4), 649-674.

13. Losada, M., \& Rodríguez, A. (2007). Calidad del servicio de salud: una revisión a la literatura desde la perspectiva del marketing. Cuadernos de Administración, 20(34), 237-258.

14. Maldonado, N., \& Tamayo, A. (28 de noviembre de 2007). Estudio integral de eficiencia de los hospitales. En Archivos de Economía(338), 64. (DNP Ed.) Colombia.

15. Mingming, L., \& Dongmei, L. (2010). An Analysis on Total Factor Productivity and Influencing Factors of Soybean in China. Journal of Agricultural Science, 2(2), 158-163.

16. Ministerio de la Protección Social. (26 de abril de 2010). Comisión de Regulación en Salud. Consulta- 
do el 27 de julio de 2010, de http://www.minproteccionsocial.gov.co/VBeContent/newsdetail. asp?id=19034\&idcompany=3

17. Ministerio de la Protección Social y Organización Panamericana de la Salud. (2008). Biblioteca Virtual para la Vigilancia en Salud Pública de Colombia. Consultado el 26 de julio de 2010, de http://www.bvsvspcol.bvsalud.org/php/level.php?lang=es\&compo nent $=22$ \&item $=132$

18. Mutis, H., Díz, G., \& Toro, E. (2006). Eficiencia técnica de hospitales nivel II en Colombia. Ingeniería y Competitividad, 8(1), 22-29.

19. Nueva EPS. (2010). Nueva EPS. Consultado el 12 de septiembre de 2010, de http://www.nuevaeps.com. co/v2/vercontenido.php?id=157

20. Ozyurt, S. (2009). Total Factor Productivity Growth in Chinese Industry: 1952-2005. Oxford Development Studies, 37(1), 1-17.

21. Pestana, C., \& Medeiros, M. (2007). Analysing the Performance of the Pension Fund Industry with a Stochastic Frontier Model: A Case Study for Portugal. Geneva Papers, 32(2), 190-210.

22. Posada, C. (6 de septiembre de 1993). Productividad, crecimiento y ciclos en la economía colombiana 1967-1992. Archivos de Macroeconomía(016), 62. (DNP, Ed.) Colombia.

23. Restrepo, J., Lopera, J., \& Rodríguez, S. (2007). La integración vertical en el sistema de salud colombiano. Revista de Economía Institucional, 9(17), 279-308.

24. Samuelson, R. (29 de marzo de 2010). Newsweek. Consultado el 26 de julio de 2010, de http://www. newsweek.com/2010/03/28/our-unhealthy-financial-future.html

25. Sánchez, F., Rodríguez, J., \& Núñez, J. (28 de agosto de 1996). Evolución y determinantes de la productividad en Colombia: un análisis global y sectorial. Archivos de Macroeconomía(050), 49. (DNP, Ed.) Colombia.
26. Sarmiento, A., Castellanos, W., Nieto, A., Alonso, C., \& Pérez, C. (6 de diciembre de 2005). Análisis de eficiencia técnica de la red pública de prestadores de servicios dentro del sistema general de seguridad social en salud. Archivos de Economía (298), 111. (DNP, Ed.) Colombia.

27. Stresing, R., Lindenberger, D., \& Kümmel, R. (2008). Cointegration of output, capital, labor, and energy. European Physical Journal B, 66(2), 279-287.

28. Varian, H. (1999). Microeconomía Intermedia. BarceIona: Antoni Bosch.

29. Vasigh, B., \& Fleming, K. (2005). A Total Factor Productivity Based Structure for Tactical Cluster Assessment: Empirical Investigation in the Airline Industry. Journal of Air Transportation, 10(1), 3-19.

30. Vinod, H. (1973). Interregional Comparison of Production Structures. Journal of Regional Science, 13(2), 261-267.

31. Wholey, D., Engberg, J., \& Bryce, C. (2006). A Descriptive Analysis of Average Productivity among Health Maintenance Organizations, 1985 to 2001. Health Care Management Science, 9(2), 189-206. 